\title{
On the driver of relativistic effect strength in Seyfert galaxies
}

\author{
M. Guainazzi ${ }^{1}$, S. Bianchi ${ }^{2,3}$, I. de la Calle Pérez ${ }^{1}$, M. Dovčiak ${ }^{4}$, and A. L. Longinotti ${ }^{5}$ \\ 1 European Space Astronomy Centre of ESA PO Box 78, Villanueva de la Cañada, 28691 Madrid, Spain \\ e-mail: Matteo.Guainazzi@sciops.esa.int \\ 2 Dipartimento di Fisica, Università degli Studi Roma Tre, via della Vasca Navale 84, 00146 Roma, Italy \\ 3 INAF - Osservatorio Astronomico di Brera, via E. Bianchi 46, 23807 Merate, Italy \\ 4 Astronomical Institute, Academy of Sciences, Boční II, 14131 Prague, Czech Republic \\ 5 MIT Kavli Institute for Astrophysics and Space Research, 77 Massachusetts Avenue, NE80-6011, Cambridge, MA 02139, USA
}

Received 1 December 2010 / Accepted 7 April 2011

\begin{abstract}
Context. Spectroscopy of X-ray emission lines emitted from accretion discs around supermassive black holes is one of the most powerful probes of the accretion flow physics and geometry, while also providing in principle observational constraints on the black hole spin. Previous studies have suggested that relativistically broadened line profiles are fairly common in nearby unobscured Seyfert galaxies. Their strength, as parametrised by the equivalent width $(E W)$ against the total underlying continuum, spans a range of almost two orders of magnitude.

Aims. We attempt to determine the ultimate physical driver of the strength of this relativistic reprocessing feature.

Methods. We first extend the hard X-ray flux-limited sample of Seyfert galaxies studied so far in the framework of the FERO (Finding Extreme Relativistic Objects) project to obscured objects up to a column density $N_{\mathrm{H}}=6 \times 10^{23} \mathrm{~cm}^{-2}$. We verify that none of the line properties depends on the AGN optical classification, as expected from the Seyfert unification scenarios. There is also no correlation between the accretion disc inclination, as derived from formal fits of the line profiles, and the optical type or host galaxy aspect angle, suggesting that the innermost regions of the accretion disc and the host galaxy plane are not aligned. We use this extended sample to study the dependence of the $E W$ on various observables, and compare it with the predictions of Monte Carlo accretion-disc reprocessing simulation.

Results. The behaviour of the $E W$ as a function of disc inclination, shape of the intrinsic power-law nuclear continuum, or iron abundance does not agree with the simulation predictions. Our observational data are not sensitive enough to the detailed ionisation state of the line-emitting disc. However, the lack of dependence of the line $E W$ on either the luminosity or the rest-frame centroid energy rules out disc ionisation playing an important role on the $E W$ dynamical range in Seyferts.

Conclusions. The dynamical range of the relativistically broadened $\mathrm{K}_{\alpha}$ iron line $E W$ in nearby Seyferts appears to be mainly determined by the properties of the innermost accretion flow. We discuss several mechanisms (disc ionisation, disc truncation, and aberration due to a mildly relativistic outflowing corona) that can explain this. We stress that the above results represent neither a falsification or proof of the relativistically blurring scenario. Observational data still do not contradict scenarios invoking different mechanisms for the spectral complexity around the iron line, most notably the "partial covering" absorption scenario.
\end{abstract}

Key words. accretion, accretion disks - relativistic processes - galaxies: nuclei - galaxies: Seyfert - X-rays: galaxies

\section{X-ray spectroscopic evidence of relativistic accretion flow in AGN}

Accretion discs feeding black holes are predicted to dissipate most of their energy in the innermost few Schwarzschild radii (Agol \& Krolik 2000; Krolik \& Hawley 2002). In these regions, the space-time distortion significantly affects the photon path in terms of general and special relativity effects (Fabian et al. 2000; Reynolds \& Novak 2003). Monochromatic lines emitted in relativistic discs are expected to suffer significant profile distortions (Fabian et al. 1989; Laor 1991). The properties of the profiles seen at infinity depend on a number of parameters related to the accretion flow (size and ionisation state of the disc photosphere), its orientation with respect to the line of sight, the shape of the illuminating ionising continuum (Matt et al. 1992; Życki et al. 1994; Nayakshin et al. 2000; Ross \& Fabian 2005), and the metal abundances. The profile in principle reflects the physical and geometrical properties of the accretion flow on spatial scales inaccessible by any other conceivable experiments in the electromagnetic domain.
For these reasons, considerable excitement followed the first detection of a broadened and skewed profile of the iron $\mathrm{K}_{\alpha}$ fluorescent iron line by ASCA in MCG-6-30-15, a nearby X-ray bright Seyfert 1 galaxy (Tanaka et al. 1995). Broad iron lines were common in ASCA spectra of AGN (Nandra et al. 1997). With the launch of major X-ray observatories at the beginning of the century - Chandra and XMM-Newton - the hope arose of being able to accumulate large samples of broad lines, which may unveil experimental clues on the way matter accretes onto supermassive black holes, as well as allow us to study its dependence on the AGN and host galaxy properties. This hope has only been partly fulfilled. Although there are plenty of measurements of broad iron $\mathrm{K}_{\alpha}$ lines (Miller 2007; as well as those of other elements or iron transitions: Mason et al. 2003; Fabian et al. 2009), there is doubt about whether many of them are unique solutions to the complexity of the AGN spectra (Reeves et al. 2004; Turner \& Miller 2009; Miller \& Turner 2010). The controversy is fierce, but this is not a topic of this paper. In the following, we assume that relativistic broadening and skewing is the correct astrophysical interpretation of the observed line profiles. If 
this assumption is ever found to be wrong, the conclusions of this paper will of course also be incorrect.

Studies of large AGN samples focusing on the properties of their relativistic lines have already been published (Nandra et al. 2007; de la Calle Pérez et al. 2010), whose conclusions can be summarised as follows:

- relativistically broadened iron $\mathrm{K}_{\alpha}$ fluorescent lines are present in $35-45 \%$ of bright nearby $(z \leq 0.01)$ Seyfert galaxies. However, taking the sensitivity limits of the available observations properly into account, the presence of a broad line with $E W>30 \mathrm{eV}$ cannot be excluded in $87 \%$ of the parent population. At even lower equivalent width $(E W)$ values, one hits the parameter space where systematic calibration uncertainties dominate;

- the line shape is consistent with it being emitted in a moderately relativistic accretion disc, as parametrised by the power-law index of the emissivity radial dependence, $\alpha(\langle\alpha\rangle=2.4 \pm 0.5)$ once emission down to the innermost circular stable orbit is assumed. In a few cases, however, the data require a significantly steeper law (notably MCG-6-30-15, Wilms et al. 2001; Fabian \& Vaughan 2003; 1H0707-495, Fabian et al. 2009; IRAS13224+3803, Ponti et al. 2010). This has been interpreted as implying that extreme light bending affects the line photon paths (Miniutti \& Fabian 2004);

- the accretion disc inclination distribution, $l$, peaks around $\simeq 30^{\circ}$. However, this might primarily be due to an observational bias against lines produced in highly inclined discs (Matt et al. 1992). Indeed, the measured disc inclination distribution is still consistent with an intrinsic random distribution (Nandra et al. 1997, 2007; de la Calle Pérez et al. 2010);

- the line $E W$ spans a range of almost one order of magnitude ( 30-300 eV). In a few cases, even larger $E W$ have been measured (4U1344-60, Piconcelli et al. 2006; AXJ04470627, Della Ceca et al. 2005: H1413+117, Chartas et al. 2007; IRAS 1334+2438, Longinotti et al. 2003: MCG-0214-009, Porquet 2006; Mkn 335, Longinotti et al. 2007; NGC 1365, Risaliti et al. 2009; PG1543+489, Vignali et al. 2008; RBS1423, Krumpe et al. 2007), which is again possibly indicative of reflection-dominated states induced by strong light bending (Miniutti \& Fabian 2004);

- the line properties and intensities are independent of the black hole mass (as the theory postulates; Fabian et al. 1989; Matt et al. 1992), luminosity, or accretion rate.

A standard plane-parallel X-ray illuminated accretion disc covering a $2 \pi$ solid angle should produce an $E W \sim 150 \mathrm{eV}$ for a typical AGN X-ray spectral shape and solar abundances (George \& Fabian 1991). The less-than-100\% detection fraction, as well as this large variety of $E W$ values need an explanation.

In this paper, we aim to investigate the ultimate physical driver of the observed broad iron line strength. For this purpose, we first extend the samples published so far, which included primarily X-ray unobscured AGN, to the whole Seyfert population up to column densities whose photoelectric cut-off does not hamper the measurement of the continuum underneath the line (Sect. 2). This allow us to investigate the correlation between the detection of a relativistically broadened iron line and both the specific AGN classification and host galaxy properties. In Sect. 3 subsequently, we perform a falsification test of the currently accepted paradigm that the main driver of the line $E W$ is the solid angle covered by the disc at the X-ray source, possibly modified by general relativity effects for the strongest lines. We discuss our results in Sect. 4.

\section{The GREDOS sample}

We extract the GREDOS (General Relativity Effects Detected in Obscured Sources) sample by correlating the 54 months 14-195 keV Swift Palermo BAT Catalogue (PBC; Cusumano et al. 2010) with the XMM-Newton observation $\log$ as of August 2010. The parent sample includes 754 AGN. The definition of the GREDOS sample is solely based on X-ray spectral properties. For a source to belong to GREDOS, the column density measured from a simple power-law continuum fit of the XMM-Newton EPIC spectra in an optimal observationdependent energy band has to be comprised between $5 \times 10^{21}$ and $6 \times 10^{23} \mathrm{~cm}^{-2}$. In reality, only one of the GREDOS objects $(1 \mathrm{ES} 0241+622)$ has got a column density $<10^{22} \mathrm{~cm}^{-2}$. The algorithm to determine this "optimal energy band" will be explained in Sect. 2.1. Moreover, we retained only sources with a BAT flux $\geq 4 \times 10^{-11} \mathrm{erg} \mathrm{cm}^{-2} \mathrm{~s}^{-1}$. This value corresponds to the count rate threshold used by de la Calle Pérez et al. (2010) to define a flux-limited sub-sample (FERO Finding Extreme Relativistic Objects) of the RXTE All Sky Survey (Revnivtsev et al. 2004).

The aforementioned upper threshold on $N_{\mathrm{H}}\left(6 \times 10^{23} \mathrm{~cm}^{-2}\right)$ was chosen to ensure that the obscuring column density does not prevent the detection of the primary emission at the energies of the iron emission features in any of the GREDOS objects.

The results for the GREDOS sample in this paper are compared to and jointly discussed with a sample extracted from the flux-limited FERO sample (de la Calle Pérez et al. 2010). The latter comprises all the flux-limited FERO AGN (21 sources) ${ }^{1}$ whose X-ray spectrum is obscured by a "cold" photoelectric column density $<5 \times 10^{21} \mathrm{~cm}^{-2}$. The FERO sub-sample defined in this way is therefore complementary to GREDOS. The FERO and GREDOS samples together can be used to test the invariance of the relativistically broadened features with the optically defined spectral type that is predicted by the Seyfert unification scenarios.

The GREDOS sample comprises 13 objects (Table 1$)^{2}$. It is important to stress that GREDOS is neither a complete nor an unbiased sub-sample of PBC. XMM-Newton observed some of the GREDOS objects multiple times. We first separately analysed the individual time-averaged spectra with simple photoelectrically absorbed power-law models. Spectra corresponding to best-fit parameter values consistent within the statistical uncertainties were merged together. For each source, we retained only the spectrum with the longest exposure time after this merging process. This is the same procedure followed for the FERO sources (de la Calle Pérez et al. 2010). In Table 2 we show the list of XMM-Newton observations IDs that were merged together.

\footnotetext{
1 Ark 120, Ark 564, ESO 198-G24, Fairall 9, H0557-385, IC 4329A, MCG-2-58-22, MCG-6-30-15, MCG+8-11-11, MR 2251178, Mrk 110, Mrk 279, Mrk 509, Mrk 766, NGC 3516, NGC 3783, NGC 4051, NGC 5548, NGC 7314, NGC 7469, and UGC 3973.

2 Two objects in GREDOS (MCG-5-23-16, NGC 526A) belong to the FERO flux-limited sample after de la Calle Pérez et al. (2010) because of the slightly different column density thresholds employed by them to define an object as "X-ray unobscured".
} 
Table 1. The GREDOS sample.

\begin{tabular}{lcccccccc}
\hline \hline Obs.\# & NED name & Type $^{a}$ & $z$ & $f_{\text {BAT }^{b}}$ & $E_{\text {soft }^{c}}$ & $r_{\mathrm{e}}{ }^{d}$ & $C_{\mathrm{t}}{ }^{e}$ & $T_{\text {exp }}{ }^{f}$ \\
\hline 0006220201 & NGC 4507 & Sy2 & 0.012 & 146.0 & 3.0 & $60 / 67$ & $0.35 / 0.5$ & $42.0 / 33.2$ \\
0067540201 & Mrk 348 & Sy2 & 0.015 & 112.0 & 2.5 & $60 / 40$ & $1 / 2$ & $26.4 / 22.5$ \\
0110930701 & NGC 4388 & Sy2 & 0.008 & 203.0 & 3.0 & $68 / 32$ & $0.5 / 1$ & $11.5 / 7.3$ \\
0144230101 & Mrk 6 & Sy1.5 & 0.019 & 43.7 & 1.0 & $85 / 37$ & $0.35 / 0.5$ & $36.0 / 31.3$ \\
0145670101 & NGC 2110 & Sy2 & 0.008 & 215.0 & 1.5 & $81 / 35$ & $0.35 / 0.5$ & $34.0 / 34.8$ \\
0152940101 & NGC 5252 & Sy2 & 0.022 & 60.1 & 2.0 & $67 / 38$ & $0.35 / 0.35$ & $41.0 / 33.3$ \\
0202860101 & NGC 7172 & Sy2 & 0.009 & 119.0 & 1.5 & $77 / 38$ & $1 / 2$ & $44.6 / 40.0$ \\
0550450301 & 1ES0241+622 & Sy1.2 & 0.045 & 51.0 & 1.0 & $70 / 40$ & $1 / 35$ & $15.2 / 12.1$ \\
0550451501 & GRS 1734-292 & Sy1 & 0.021 & 103.0 & 1.0 & $112 / 38$ & $1 / 0.5$ & $17.2 / 13.5$ \\
Table 2 & NGC 5506 & Sy2 & 0.006 & 196.0 & 2.0 & $50 / 50$ & $0.35 / 0.35$ & $61.0 / 117.8$ \\
Table 2 & NGC 4151 & Sy1.5 & 0.003 & 376.0 & 2.2 & $38 / 39$ & $0.35 / 0.5$ & $87.5 / 71.9$ \\
Table 2 & NGC 526A & Sy1.9 & 0.019 & 42.6 & 1.2 & $89 / 38$ & $0.35 / 0.5$ & $53.7 / 45.4$ \\
Table 2 & MCG-05-23-016 & Sy2 & 0.008 & 166.0 & 1.8 & $40 / 47$ & $0.35 / 0.5$ & $118.4 / 104.8$ \\
\hline
\end{tabular}

Notes. ${ }^{(a)}$ Optical Seyfert type according to the Véron-Cetty \& Véron catalogue (Véron-Cetty \& Véron 2010). ${ }^{(b)}$ The $14-195$ keV BAT flux (in units of $10^{-12} \mathrm{erg} \mathrm{cm}^{-2} \mathrm{~s}^{-1}$ ) after Cusumano et al. (2010). ${ }^{(c)}$ Low boundary of the energy interval where the spectral fit was performed. ${ }^{(d)}$ Size (in arc-seconds) of the source spectrum extraction region (MOS/pn). ${ }^{(e)}$ Threshold (in counts per second) on the single-event, high-energy light curves to reject intervals of high particle background (MOS/pn). ${ }^{(f)}$ Net exposure time in ks (MOS/pn) after data screening.

Table 2. List of merged observations for GREDOS sources.

\begin{tabular}{lc}
\hline \hline Source & Merged Obs.\# \\
\hline NGC 4151 & 0112310101 0112830201, 0112830501 \\
NGC 526A & 01509401010109130201 \\
NGC 5506 & 0554170101,0554170201 \\
MCG-5-23-16 & 0112830301,0302850201 \\
\hline
\end{tabular}

\subsection{Data reduction and analysis}

We discuss the data taken with the EPIC cameras only: EPIC-pn (Strüder et al. 2001), and EPIC/MOS (Turner et al. 2001). Data were reduced using SASv10 (Gabriel et al. 2003) using the calibration files available in August 2010. Calibrated event lists were generated using the reduction meta-tasks e [mp]proc. Source spectra were extracted from circular extraction regions centred on the source X-ray centroid. The size of the extraction region $r_{\mathrm{e}}$ for the source scientific products, as well as the count rate thresholds $C_{\mathrm{t}}$ employed to reject intervals of high particle background were determined by using an iterative process to maximise the net source spectra signal-to-noise ratio (see Table 1). To measure the particle-induced background, we used high-energy (MOS: $E>10 \mathrm{keV}$; pn: $10 \mathrm{keV} \leq E \leq 12 \mathrm{keV}$ ) for the whole field-ofview, light curve binned to $\Delta t=10 \mathrm{~s}$. Background spectra were extracted from off-axis circular regions on the same CCD as the source, and additionally at the same RAWY position in the EPICpn camera to ensure that the same charge transfer efficiency correction applies, as this correction depends on the distance from the readout node. Response files for each spectrum were generated using the SAS tasks arfgen and rmfgen.

Spectra were rebinned to ensure that: a) each backgroundsubtracted spectral channel contains at least 50 counts; b) the instrumental intrinsic resolution is oversampled by a factor of no larger than three. These conditions ensure that we can use the $\chi^{2}$ as a goodness-of-fit test in the forthcoming spectral analysis. Spectral fits were performed in the energy range $\left(E_{\mathrm{soft}}\right.$, $10.0 \mathrm{keV}$ ), where $E_{\text {soft }}$ (see Table 1) was determined for each spectrum after visual inspection as the energy at which excess emission above the extrapolation of the photoelectrically absorbed power-law emission begins to dominate. The rationale behind this choice is driven by the empirical evidence that this soft excess is associated with diffuse emission extended on scales as large as a few kilo-parsecs, most likely due to gas associated with the narrow and extended narrow line regions (Bianchi et al. 2006), with contributions of strong starburst emission in a few objects (Guainazzi et al. 2009). The modelling of this component is therefore fully decoupled from that of the the primary nuclear emission.

In this paper, we quote statistical errors at the $90 \%$ confidence level for one interesting parameter unless otherwise specified. To calculate luminosities, we use the cosmological parameters $H_{0}=70 \mathrm{~km} \mathrm{~s}^{-1} \mathrm{Mpc}^{-1}, \Lambda_{0}=0.73$, and $\Omega_{\mathrm{M}}=0.27$ (Bennett et al. 2003).

\subsection{Spectral model definition}

The main feature characterising X-ray spectra of obscured AGN is a soft X-ray photoelectric cut-off caused by the high column densities of obscuring gas (Awaki et al. 1991). We therefore first fit the GREDOS spectra with a simple baseline continuum constituted by a power-law modified by photoelectric absorption through cold matter. However, moderate-resolution spectroscopy of nearby Seyfert galaxies identifies complex spectral behaviour, associated with reprocessing of the primary nuclear continuum (Pounds et al. 1990; Nandra \& Pounds 1994; Turner et al. 1997). This evidence led us to add to the model continuum a Compton-reflection component (model pexrav in Xspec; Magdziarz \& Zdziarski 1995), as well as a number of Gaussian emission line profiles to account for the $\mathrm{K}_{\alpha}$ (with its Compton shoulder; Matt 2002) and $\mathrm{K}_{\beta}$ fluorescent lines from neutral iron (Turner et al. 1997), and for recombination lines from He- and H-like iron (Netzer et al. 1998; Bianchi \& Matt 2002). This "non-relativistic" baseline model can be described as follows

$$
\begin{aligned}
M(E)= & \mathrm{e}^{-\sigma_{\mathrm{p}}(E) N_{\mathrm{H}}} \times\left[A_{1} E^{-\Gamma}+\Sigma_{i} N_{i} G_{i}\left(E_{i}\right)\right. \\
& \left.+A_{2} C_{S}(E)\right]+A_{3} C_{R}(E, \Gamma),
\end{aligned}
$$

where $\sigma_{\mathrm{p}}$ is the photoelectric cross-section, $G_{i}$ are Gaussian profiles, $C_{\mathrm{S}}$ is the Compton shoulder, $C_{\mathrm{R}}$ is the Compton reflection continuum, and $A_{i}$ and $N_{i}$ are normalisation constants. We used the ztbabs implementation of the photoelectric absorption in XsPec. In the baseline, as well as all subsequent more complex models, we made the following assumptions:

- the photon index $\Gamma$ of the power law and the Compton reflection continuum has been constrained to be identical in value; 
Table 3. Summary of spectral fit results for the GREDOS sample: continuum components.

\begin{tabular}{lccccccc}
\hline \hline Source & $\Gamma$ & $\begin{array}{c}N_{\mathrm{H}, 1} \\
\left(10^{22} \mathrm{~cm}^{-2}\right)\end{array}$ & $\begin{array}{c}N_{\mathrm{H}, 2} \\
\left(10^{22} \mathrm{~cm}^{-2}\right)\end{array}$ & $\log (\xi) / C_{f}{ }^{a}$ & $R_{\mathrm{nr}}{ }^{b}$ & $R_{r}{ }^{c}$ & Abs. Model $^{d}$ \\
\hline NGC 4507 & $1.57 \pm_{0.14}^{0.07}$ & $58 \pm_{5}^{41} d$ & $7 \pm 3$ & $<2.1$ & $0.55 \pm_{0.06}^{0.14}$ & $<0.1$ & $\mathrm{CW}$ \\
NGC 4388 & $1.86 \pm_{0.35}^{0.03}$ & $21.2 \pm_{0.5}^{0.9}$ & $4 \pm 3$ & $2.0 \pm_{0.2}^{0.7}$ & $0.8 \pm 0.3$ & $0.26 \pm_{0.06}^{0.42}$ & $\mathrm{CW}$ \\
Mrk 6 & $1.43 \pm_{0.07}^{0.05}$ & $1.57 \pm_{0.12}^{0.13}$ & $\ldots$ & $0.85 \pm 0.02$ & $<0.4$ & $<2.9$ & $\mathrm{PC}$ \\
NGC 2110 & $1.69 \pm_{0.02}^{0.03}$ & $2.89 \pm_{0.36}^{0.12}$ & $9.3 \pm_{2.1}^{1.0}$ & $\ldots$ & $0.9 \pm 0.5$ & $3.4 \pm 0.7$ & $\mathrm{DC}$ \\
NGC 5252 & $1.56 \pm_{0.19}^{0.32}$ & $3.5 \pm_{1.3}^{0.9}$ & $16 \pm_{2}^{25}$ & $\ldots$ & $<0.1$ & 0.4 & $\mathrm{DC}$ \\
NGC 7172 & $1.63 \pm_{0.02}^{0.06}$ & $13.3 \pm 0.2$ & $\ldots$ & $\ldots$ & $<0.1$ & $0.68 \pm_{0.03}^{0.10}$ & $\mathrm{SC}$ \\
1ES0241+622 & $1.61 \pm_{0.04}^{0.09}$ & $0.46 \pm_{0.04}^{0.07}$ & $\ldots$ & $\ldots$ & $<0.6$ & $<0.1$ & $\mathrm{SC}$ \\
GRS1734-292 & $1.41 \pm_{0.02}^{0.03}$ & $1.41 \pm_{0.02}$ & $\ldots$ & $\ldots$ & $<0.1$ & $<0.4$ & $\mathrm{SC}$ \\
NGC 5506 & $1.689 \pm_{0.013}^{0.011}$ & $3.4 \pm_{0.5}^{0.7}$ & $1.5 \pm_{0.8}^{0.5}$ & $<-0.27$ & $<0.1$ & $<0.1$ & $\mathrm{CW}$ \\
NGC 4151 & $1.446 \pm_{0.009}^{0.010}$ & $13.21 \pm_{0.25}^{0.14}$ & $\ldots$ & $0.853 \pm 0.004$ & $1.07 \pm_{0.56}^{0.09}$ & $<0.1$ & $\mathrm{PC}$ \\
NGC 526A & $1.457 \pm_{0.013}^{0.021}$ & $1.56 \pm_{0.03}^{0.04}$ & $\ldots$ & $\ldots$ & $<0.06$ & $<0.12$ & $\mathrm{SC}$ \\
MCG-05-23-016 & $1.632 \pm_{0.016}^{0.010}$ & $1.849 \pm_{0.040}^{0.014}$ & $\ldots$ & $\ldots$ & $<0.64$ & $<0.10$ & $\mathrm{SC}$ \\
\hline
\end{tabular}

Notes. ${ }^{(a)}$ Ionisation parameter for the warm absorber component for model CW or covering fraction of the patchy absorber for model PC. ${ }^{(b)}$ Ratio of the normalisation of the non-relativistic reflection component to the primary power-law. ${ }^{(c)}$ Ratio of the normalisation of the relativistic reflection component to the primary power-law. ${ }^{(d)}$ Absorption model. Legenda: $\mathrm{SC}=$ single cold absorber; $\mathrm{DC}=$ double cold absorber; $\mathrm{PC}=$ partial covering; $\mathrm{CW}=$ cold and warm absorber.

- the power law is modified by a high-energy cut-off beyond the energy bandpass of the EPIC cameras. It was fixed at $150 \mathrm{keV}$ (Risaliti 2002);

- the inclination of the Compton reflection component with respect to the line-of-sight was kept fixed at $45^{\circ}$. This parameter is degenerate with the relative normalisation between the Compton reflection and the primary continuum, hence this assumption does not unnecessarily constrain the parameter space of other spectral parameters;

- we assumed solar abundances according to Anders \& Grevesse (1989);

- the centroid energy of the Gaussian profiles was fixed at values as dictated by the atomic physics, i.e. $6.4 \mathrm{keV}, 6.966 \mathrm{keV}$, and $7.058 \mathrm{keV}$ for $\mathrm{FE}$ I $\mathrm{K}_{\alpha}$, FE XxvI, and FE I $\mathrm{K}_{\beta}$, respectively. The intensity of the $\mathrm{FE}$ I $\mathrm{K}_{\beta}$ was constrained such that it did not exceed $16 \%$ of the $\mathrm{K}_{\alpha}$ intensity. For the FE Xxv transitions, we allowed the centroid energy to vary between $6.6364 \mathrm{keV}$ and $6.7000 \mathrm{keV}$, corresponding to the forbidden and the resonant transitions, respectively;

- the intrinsic width of the Gaussian profiles was assumed to be the same for a given object, and consistent with the instrumental resolution;

- following Matt (2002), the Compton shoulder was modelled with a rectangular box of width $50 \mathrm{eV}$ and intensity that had been constrained so as not to exceed $20 \%$ of the intensity of the corresponding $\mathrm{K}_{\alpha}$ profile.

Only after these additional components were included (and then retained) if their inclusion improved the quality of the fit at a confidence level $>90 \%$; Lampton et al. 1976), we tried to fit any additional spectral complexity with relativistically blurred disc components. For this scope, we used models extracted from the ky suite (Dovčiak et al. 2004), based on a common ray-tracing subroutine developed to describe the X-ray emission of blackhole accretion discs in the strong gravity regime. Since any emission line should be accompanied by a relativistically blurred disc continuum, we used a combination of the models kyrline and pexrav, where the latter was convolved with a relativistic kernel kyconv. In both kyrline and kyconv, the blurring effect depends on the following parameters: a) the black hole spin $a$ (comprised between 0 and 0.9982 in dimensionless relativistic units); b) the inner $\left(r_{\text {in }}\right)$ and outer $\left(r_{\text {out }}\right)$ radius of an annular region of the disc from which the line photons are emitted; c) the index $\alpha$ of the radial dependence of the emissivity per unit area $\kappa$, in the local frame comoving with the disc: $\kappa(r) \propto r^{-\alpha}$; d) the "disc inclination" $l$, i.e., the angle between the normal to the disc plane and the line-of-sight; and e) the rest-frame energy (assumed monochromatic) of the photons emitted by the disc, $E_{\mathrm{c}}$. Although the line normalisation in kyrline is expressed in units of photons $\mathrm{cm}^{-2} \mathrm{~s}^{-1}$ integrated over the whole profile, we also use the line $E W$, i.e., the line intensity normalised to the underlying continuum at $6.4 \mathrm{keV}$, to ease comparison with theoretical predictions. Following the approach described in Guainazzi et al. (2010), we fixed the values of $r_{\text {in }}$ and $r_{\text {out }}$ to the innermost circular stable orbit (e.g. $r_{\text {in }}=1.24 r_{\mathrm{g}}$ for a maximally spinning black hole) and $r_{\text {out }}=400 r_{\mathrm{g}}$, respectively. Constraints on the black hole spin could not be derived for any of the GREDOS sources. We therefore assumed a maximally spinning black hole in all the subsequent fits.

For objects where this model did not provide an adequate fit, we changed the obscuration law. We tested four additional prescriptions for the X-ray obscuration: a) two "cold" absorbing systems, one covering the primary and the relativistically broadened reprocessing components, and the other covering the whole model; b) a partial covering absorber that obscures solely the primary and the relativistically broadened reprocessing components; c) one ionised absorber, calculated according to a grid of CLoudy models (Ferland et al. 1998) as described in Bianchi et al. (2010); and d) the combination of a "cold" and a "ionised" absorber.

For each source, we selected the model that corresponds to the smallest value of reduced $\chi^{2}$. We consider in the following a relativistic line as detected if the variation of $\chi^{2}$ associated with the inclusion of the kyrline component in the spectral model is significant at the 5- $\sigma$ level, to be consistent with the criterion adopted by de la Calle Pérez et al. (2010).

\subsection{Spectral results}

Tables 3 and 4 in Appendix A summarise the results of our spectral fits for the GREDOS sample. At the adopted 5- $\sigma$ confidence level, a broadened relativistic line is detected in only two sources. In both cases, this detection has been reported 


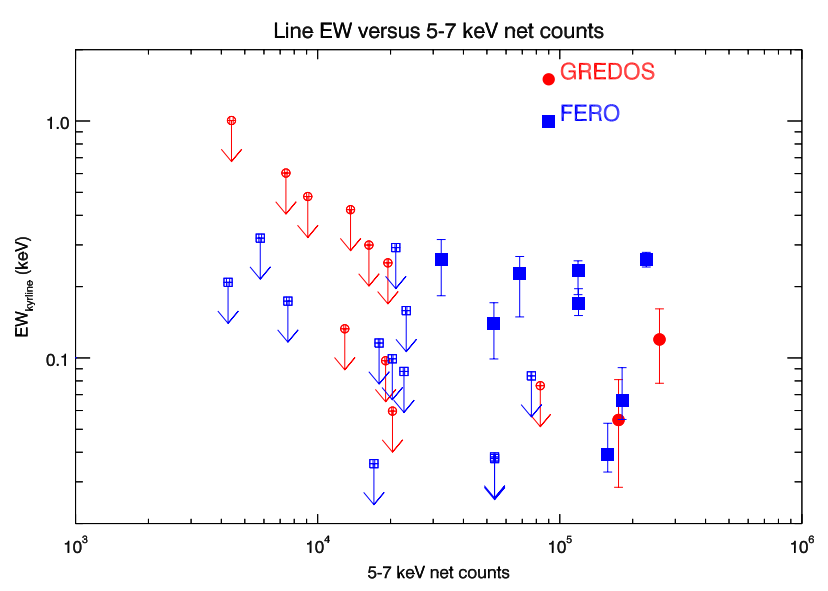

Fig. 1. Relativistic line $E W$ as a function of the background-subtracted 5-7 keV counts in the GREDOS (circles) and FERO (squared) samples sources. Empty symbols indicate upper limits. The lines represent the sample sensitivity for three different values of inclination angle, assuming $\alpha=2.5$ : dashed line: $10^{\circ} ;$ dot-dashed line: $40^{\circ}$; dot-dot-dashed line: $80^{\circ}$.

elsewhere: MCG-5-23-16 (Dewangan et al. 2003; Reeves et al. 2007; de la Calle Pérez et al. 2010), and NGC 5506 (Guainazzi et al. 2010). Our non-detections are also consistent with previously published results: Mkn 6 (Schurch et al. 2006), NGC 4151 (Schurch et al. 2003), NGC 4388 (Beckmann et al. 2004), and NGC 5252 (Dadina et al. 2010). The detection fraction in GREDOS $(\simeq 15 \%)$ is nominally smaller than for unobscured AGN. However, the GREDOS sample is incomplete. This prevents a statistically robust comparison of this number with less unbiased samples such as FERO. Moreover, most of the GREDOS sources are underexposed. In Fig. 1, the $E W$ of the relativistic line is plotted against the background-subtracted counts in the $5-7 \mathrm{keV}$ energy band. As already observed by other authors (Guainazzi et al. 2006; de la Calle Pérez et al. 2010), the detection is a strong function of the number of net source counts in the hard X-ray band. All broad-line detections in the FERO and in the GREDOS samples correspond to objects with more then 30000 net source counts in the 5-7 keV energy band. Only three sources in GREDOS satisfy this empirical criterion: MCG5-23-16, NGC 5506 (both of its detections), and NGC 4151. Above 100000 net counts, one measures interestingly a $100 \%$ detection fraction.

At a lower confidence level $(3 \sigma)$, an additional broad line is detected in the GREDOS source NGC 4507, in contrast to the finding of Matt et al. (2004). Its parameters are $\alpha \leq 1.7$, $l=10 \pm_{8}^{4 \circ}$, and $E W=128 \pm_{60}^{4} \mathrm{eV}$ if $E_{\mathrm{c}} \equiv 6.4 \mathrm{keV}$. We do not include this measurement in the joint analysis of the FERO and GREDOS detections (see Sect. 2.4). The profiles of the iron lines in GREDOS are shown in Fig. 2. These line profiles are the ratio of data to the best-fit model after removal of the relativistic line profile (kyrline component). In Fig. 3, we show the fractional contribution of the continuum as well as the narrow-band emission features to the total best-fit model in the two GREDOS objects, where a broad line is detected at a confidence level $\geq 5 \sigma$.

\subsection{FERO+GREDOS detections}

Hereafter we consider the detections in the FERO and GREDOS samples jointly (cf. Sect. 2 for the definition of the FERO sample in this paper). In this sample, 12 broad iron lines are detected at confidence level $\geq 5 \sigma$ (cf. Table 2 in de la Calle Pérez et al. 2010; and Table 4 in this paper).

Once the FERO and GREDOS detections are considered jointly, neither the detection fraction nor the line $E W$ depend on the optical type (Fig. 4), despite there being a nominal trend for broad lines to be more common in "type 1" Seyferts. In this paper, we use the optical type as defined in the Veron-Cetty \& Veron (2010) catalogue. We use the optical classification to place, to the best of our knowledge, the FERO+GREDOS sources in the framework of the standard Seyfert unification scenario as reviewed in, e.g., Antonucci (1993). For this purpose, objects that exhibit broad polarised Balmer lines and/or weak IR broad lines, but whose optical spectrum is typical of obscured AGN are still considered to be "type 2"3. For sake of simplicity we include Veron-type 1.9 objects into the "type 2" class, Veron type 1.2 objects, and narrow-line Seyfert galaxies ${ }^{4}$ into the "type 1" class, hereafter.

Following the same approach as in, e.g., Guainazzi et al. (2010), we use the $\alpha$ parameter in kyrline as a measurement of the importance of relativistic effects in shaping the profile of the iron $\mathrm{K}_{\alpha}$ emission line. When the inner and outer radius of the line-emitting region are equal, higher $\alpha$ values indicate that line photons are emitted in regions closer to innermost stable orbit of the accretion disc. For $\alpha \leq 2$ (i.e., when a large fraction of the accretion disc is responsible for the line production), the choice of the line-emitting-region outer radius (which, we recall, is fixed to be $400 r_{\mathrm{g}}$ in our spectral models) may affect the best-fit values of other line parameters. This effect is discussed by Guainazzi et al. (2010) for NGC 5506.

For the joint FERO and GREDOS detections, one observes an apparent correlation between $\alpha$ and $E W$ (Fig. 5). A fit to the data using an extension of the regression method on censored data originally described by Schmitt (1985) and Isobe et al. (1986) yields $\alpha=(0.44 \pm 0.19)+(2.0 \pm 0.8) \times \log (E W)$, where $E W$ is expressed in $\mathrm{keV}$. The Spearmann rank coefficient for the same data yields a correlation probability of $99.7 \pm_{17.4}^{0.2} \%$, where the nominal value is calculated using only bracketed measurements, and the error bars reflect the range of values that censored data could cover on the $y$-axis (only subject to the bestfit model parameter restriction $\alpha \leq 6$ ). Taking into account the large uncertainties, the correlation is statistically marginal. In the same figure, we compare the measurements with the predictions of an axisymmetric lamppost model (Dovčiak et al. 2004), where a point-like source of X-ray radiation is located at variable heights along the black hole spin axis. We simulated EPICpn spectra according to this model, and fit them using the same baseline model as defined in Sect. 2.2. Moderately relativistic $(\alpha \lesssim 3)$ lines can be explained in this framework if the accretion disc is seen in different objects of a wide range of different inclination angles and heights. Interestingly, it is impossible for $\alpha>3$ in this scenario. We note that examples of extreme relativistic lines have been published, whose profiles are consistent with very steep ( $\alpha \gtrsim 5)$ specific emissivity radial dependences (Wilms et al. 2001; Fabian et al. 2009; Ponti et al. 2010). The main driver of some of these results is the very smooth shape of the soft excess, implying extreme relativistic blurring of narrow-band spectral features imprinted by disc reflection on the soft X-ray $(E \lesssim 2 \mathrm{keV})$ spectrum. As pointed out by, e.g., Życki et al. (2010), steep profiles can be obtained only if the X-ray source is not located on the black-hole spin axis, because they

3 MCG-5-23-16, Mkn 348, NGC 2110, NGC 4388, NGC 4507, NGC 5506, NGC 7314.

${ }^{4}$ Mkn 110, Mkn 766, NGC 4051. 

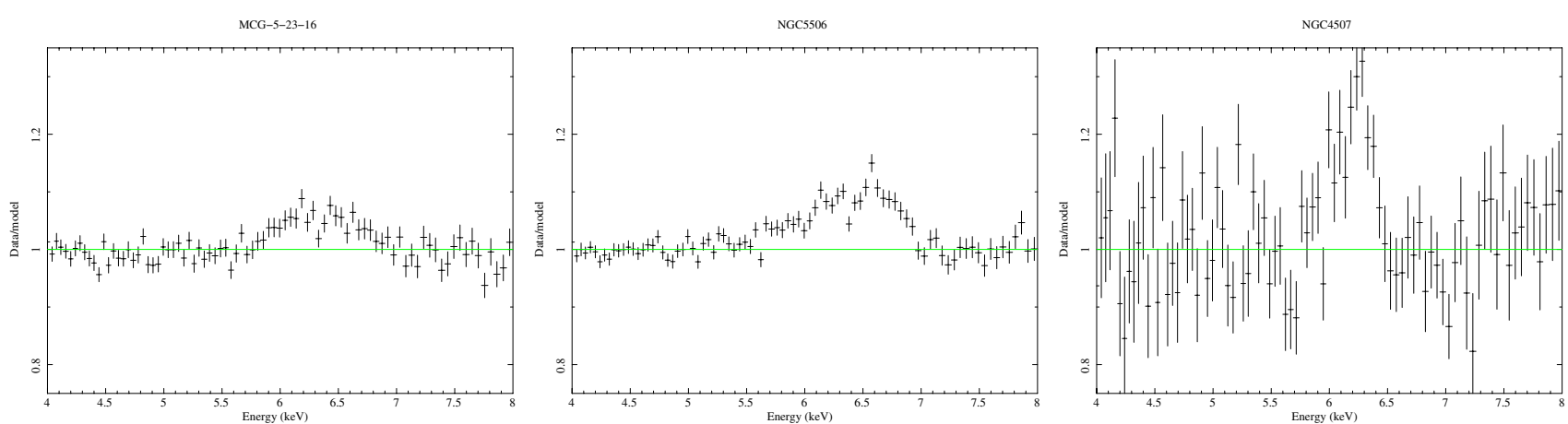

Fig. 2. Profiles of the relativistically broadened iron lines detected in the GREDOS sample. They represent ratios of the data to the model once the kyrline component is removed from the best-fit model. The lines in MCG-5-23-16 and NGC 5506 are detected at a confidence level $\geq 5 \sigma$ in the relativistically blurred line scenario. The line in NGC 4507 is detected at a confidence level $\simeq 3 \sigma$ only.
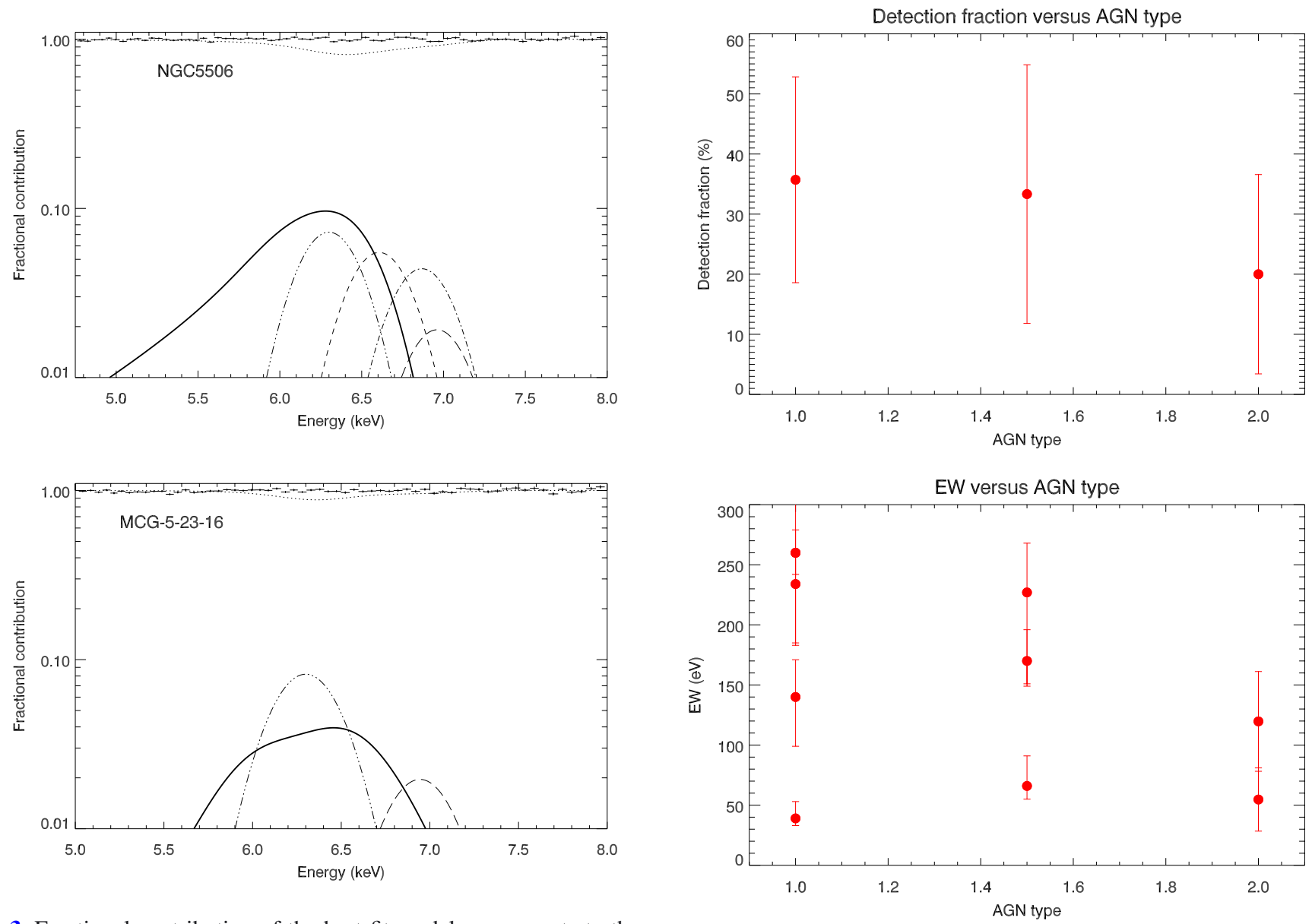

Fig. 3. Fractional contribution of the best-fit model components to the total best-fit model. Dotted line: continuum; solid line: relativistically broadened $\mathrm{Fe}$; dashed-multi-dotted line: unresolved $\mathrm{Fe} \mathrm{K}_{\alpha}$; dashed line: unresolved Fexxv recombination; dashed-dotted line: unresolved Fexxvi recombination; dashed line: unresolved $\mathrm{Fe}$ I $\mathrm{K}_{\beta}$ line. All components have been smoothed with a Gaussian kernel $\left(\sigma_{\text {kernel }}=150 \mathrm{eV}\right)$ to reproduce the effect of the EPIC-pn intrinsic energy resolution. The crosses represents the data/model ratio against the best-fit model.

require the combination of dynamical Doppler shift and frame dragging within the black hole ergosphere.

There is no significant difference between the distribution of disc inclination angles between the GREDOS and the FERO sample sources, nor any clearly evident dependence of the disc inclination angle on the optical spectral type (Fig. 6) or host

Fig. 4. Relativistic line detection fraction (upper panel) and $E W$ (lower panel) as a function of optical type for the sources of joint FERO+GREDOS samples.

galaxy inclination. Although the line detections in a type 2 Seyfert correspond to $l \geq 38^{\circ}$ (MCG-5-23-16, Reeves et al. 2007; NGC 5506, Guainazzi et al. 2010), high-inclination lines are measured in type 1 objects as well (Mkn 509, MCG-6-3015; de la Calle Pérez et al. 2010). Moreover, type 1.5 objects invariably exhibit inclination angles smaller than $40^{\circ}$, ruling out a simple relation between the inclination of the nuclear obscuring matter (as measured by the optical type) and that of the accreting matter. 


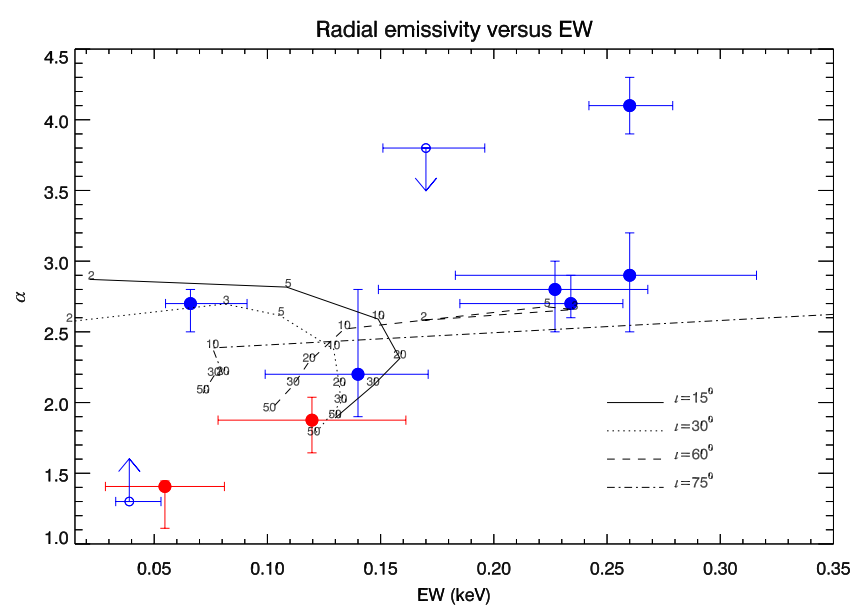

Fig. 5. $\alpha$ versus $E W$ for the iron $\mathrm{K}_{\alpha}$ relativistic lines detected in the FERO and GREDOS samples. Empty symbols indicate upper limits. The lines represent the predictions of a lamppost model for different values of the X-ray source height (small numbers along the curves in units of gravitational radii) and disc inclinations (line styles; cf. the inset label).

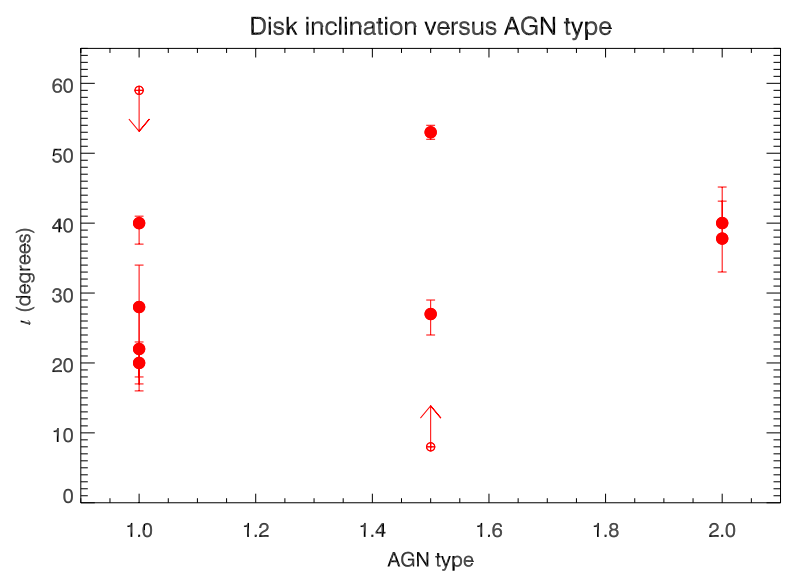

Fig. 6. Accretion disc inclination angle $\imath$ as a function of the optical type for the joint GREDOS and FERO control sample discussed in this paper.

Care must be taken when interpreting direct measurements of the disc inclination using the profile of a relativistically broadened line, though. Measurements of the inclination angles determined using X-ray spectroscopy in the relativistically blurredline scenario are still affected by large systematic uncertainties. Even in the most well-studied case of the high-quality "longlook" EPIC spectrum of MCG-6-30-15 taken in 2002, published measurements of the relativistic line profiles by different authors yield values of the disc inclination angle ranging from $20^{\circ}$ to $48^{\circ}$ (Brenneman \& Reynolds 2006; Fabian et al. 2002; Branduardi-Raymont et al. 2001; Reynolds et al. 2004).

\section{The quest for the ultimate driver of relativistic effect strength}

A number of AGN exhibit relativistically broadened iron lines with an $E W \geq 300 \mathrm{eV}$ (cf. Sect. 1). This extreme strength is difficult to reconcile with theoretical calculations of the $E W$ expected from standard X-ray illuminated relativistic accretion discs (George \& Fabian 1991), unless the
Table 4. Summary of spectral fit results for the GREDOS sample: relativistic line.

\begin{tabular}{lccc}
\hline \hline Source & $\begin{array}{c}l \\
\left({ }^{\circ}\right)\end{array}$ & $\alpha$ & $\begin{array}{c}E W \\
(\mathrm{eV})\end{array}$ \\
\hline NGC 4507 & $\ldots$ & $\ldots$ & $<130$ \\
Mrk 348 & $\ldots$ & $\ldots$ & $<300$ \\
NGC 4388 & $\ldots$ & $\ldots$ & $\ldots{ }^{a}$ \\
Mrk 6 & $\ldots$ & $\ldots$ & $<480$ \\
NGC 2110 & $\ldots$ & $\ldots$ & $<100$ \\
NGC 5252 & $\ldots$ & $\ldots$ & $<600$ \\
NGC 7172 & $\ldots$ & $\ldots$ & $<60$ \\
1ES0241+622 & $\ldots$ & $\ldots$ & $<1000$ \\
GRS1734-292 & $\ldots$ & $\ldots$ & $<420$ \\
NGC 5506 & $40.0 \pm_{1.9}^{3.1}$ & $1.88 \pm_{0.23}^{0.16}$ & $120 \pm 40$ \\
NGC 4151 & $\ldots$ & $\ldots$ & $<80$ \\
NGC 526 & $\ldots$ & $\ldots$ & $<250$ \\
MCG-05-23-016 & $38 \pm_{5}^{7}$ & $1.41 \pm_{0.30}^{0.04}$ & $55 \pm 26$ \\
\hline
\end{tabular}

Notes. All measurements refer to a rest-frame centroid energy $E_{\mathrm{c}}=$ $6.4 \mathrm{keV}$. Upper limits are at the $90 \%$ level. ${ }^{(a)}$ Unconstrained.

primary emission illuminating the disc is highly anisotropic. Mechanisms based on strong relativistic effects occurring when the source is located a few gravitational radii from the black hole event horizon have been proposed (Martocchia et al. 2002; Miniutti \& Fabian 2004). Light bending coupled with a variable height of the X-ray primary sources above the disc plane can explain at the same time the extreme strength of relativistic reprocessing spectral components (Fabian et al. 2009; Ponti et al. 2010), and their lack of response to the primary continuum variation (Miniutti \& Fabian 2004).

A standard plane-parallel X-ray illuminated accretion disc covering a $2 \pi$ solid angle should produce an $E W \sim 150 \mathrm{eV}$ for a typical AGN X-ray spectral shape and solar abundances (George \& Fabian 1991). However, many FERO+GREDOS sources exhibit $E W$ s of significantly smaller value. Are there alternatives to explaining this large dynamical range in terms of disk solid angle as seen by the primary source? We hereby follow a falsification approach to addressing this problem: we assume that for "standard" $E W \leq 300 \mathrm{eV} \mathrm{AGN}$, the solid angle is on average the same, and analyse the consequences that this assumption bears on the correlation between $E W$ and other observables.

We first introduce the most fundamental limitation of our study. High- $E W$ reprocessed emission lines can be produced when the disc photosphere is highly ionised (Życki et al. 1994). The data currently do not allow us to test this hypothesis. Fits with a relativistic profile corresponding to being dominated by Fe He-like emission is preferred in 3 out of 11 FERO (de la Calle Pérez et al. 2010), in none of the GREDOS detections. Although in these cases the $E W$ is tendentiously larger then the distribution average (140-230 eV), this parameter space is not exclusively occupied by them. Moreover, there is no correlation between the line $E W$ and either the X-ray luminosity or the accretion rate normalised to the Eddington value. This suggests that disc photoionisation probably does not play a decisive role in determining the strength of relativistic reprocessing for our sample. Much higher data quality would be required, however, to resolve the degeneracy between the bulk ionisation state of the line emitting region and other parameters. 
Once we neglect the disc ionisation, the $E W$ of a relativistically broadened iron line depends primarily on:

1. the photon index of the illuminating continuum, $\Gamma$;

2. the disc inclination, $l$;

3. the iron abundance, $Z_{\mathrm{Fe}}$;

4. the "reflection fraction", a parameter that for purely isotropic emission is proportional to the solid angle subtended by the disc at the X-ray source.

Approximate formulae for the dependence on the first three parameters are reported by Nandra et al. $(2007)^{5}$. In this section we initially assume that the reflection fraction is the same for all objects in the FERO+GREDOS sample and test whether the observed distribution of $E W$ can be reproduced by any of the other three parameters.

Very few measurements of the iron abundance are available in the literature for the objects of our sample. Following the approach of Ballantyne (2010), we therefore decided to calculate $Z_{\mathrm{Fe}}$ using the correlation with the accretion rate in Netzer \& Trakhtenbrot (2007).

In Fig. 7, we show the relation between the $E W$ of the relativistically broadened iron line and $\Gamma, l$, and $Z$ for two samples:

1. the full FERO+GREDOS sample (left panels);

2 . the 12 FERO+GREDOS detections only, once the $E W$ values have been corrected for the dependence of the $E W$ on the other two parameters assuming as common reference the values: $\Gamma_{0}=1.9, \iota_{0}=30^{\circ}$, and $Z_{\mathrm{Fe}, 0}=Z_{\odot}$.

The observed trends of $E W$ against any of the above parameters do not agree with the theoretical predictions. The correlation with the metallicity fails to reproduce the whole dynamical range covered by the $E W$ measurements for $Z_{\mathrm{Fe}} \geq 0.5 Z_{\odot}$.

Figure 7 demonstrates that the falsification tests we performed fails. We are left with the conclusion that the "reflection fraction" is the main driver of the relativistically broadened iron line $E W$ even when X-ray spectra are not disc-reflection dominated. In Fig. 8, we show the correlation between the $E W$ and the parameter $R$, which is usually used to characterise quantitatively the reflection fraction. A similar correlation is found between $\alpha$ and $R$, as expected given the tentative correlation between $\alpha$ and $E W$ shown in Fig. 5. We note that $R$ is proportional to the solid angle, $\Omega$, subtended by the disc at the X-ray isotropic source, and $R=1$ for $\Omega=2 \pi$. Unfortunately, the scientific payload on-board XMM-newton does not allow the simultaneous measurement of $R$. We therefore derived $R$ from literature nonsimultaneous measurements. In most cases, we used the results of the BeppoSAX survey of AGN presented in Dadina (2007), except for those objects for which more recent measurements by Suzaku are available: NGC 3516 (Markowitz et al. 2008), and IC4329A (Markowitz et al. 2006). With these data, the measured values of $R$ is primarily driven by the strength of the hard ( $E \geq 10 \mathrm{keV}$ ) excess above the extrapolation of the primary continuum. These measurements therefore assume that the hard X-ray excess is dominated by reflection. In some cases (Turner \& Miller 2009; Reeves et al. 2009), it appears that absorption may play a greater role (see also Walton et al. 2010).

Care has to be exercised in interpreting this plot, because of the lack of simultaneity of the measurements, as well as in considering the different astrophysical models used to fit

\footnotetext{
5 The formula for the dependence of the $E W$ on the spectral index contains a typo. The correct formula is $E W=E W_{0} \times\left[9.66 \times\left(\Gamma^{-2.80}\right)-0.56\right]$ (Nandra, priv. comm.), where $E W_{0}=144 \mathrm{eV}$.
}

the XMM-Newton/EPIC, the Suzaku, and the BeppoSAX data. Moreover, a contribution to the continuum Compton-reflection certainly comes from optically thick matter on pc-scales, which are responsible for the bulk of the unresolved component of the narrow $\mathrm{K}_{\alpha}$ iron line (Page et al. 2004; Bianchi et al. 2007; Shu et al. 2010). Bearing these caveats in mind, close agreement between $R$ and $E W$ is found, as already reported by previous authors (Perola et al. 2002; Dadina 2008; Walton et al. 2010). On the basis of broadband X-ray measurements, it has been proposed that a transmitted component seen through a partial covered absorber could contribute to the emission above $10 \mathrm{keV}$, which is crucial for the measurement of the reflection fraction (Turner et al. 2009). Studies of sizable samples of bright AGN with missions ensuring a broadband simultaneous coverage, such as Suzaku or the future Astro-H and Astrosat, would be very valuable in this respect.

\section{Conclusions}

In this paper, we have tried to address why the $E W$ of the relativistically broadened $\mathrm{K}_{\alpha}$ fluorescent iron line in AGN covers a dynamical range of almost two orders or magnitude if the standard theory (reflection of the X-ray isotropic primary emission by a plane-parallel infinite accretion disc) predicts variations by at most a factor of a few?

In a few cases of very strong $(E W>300 \mathrm{eV})$ lines, that a "reflection dominated" scenario applies seems inescapable (Miniutti \& Fabian 2004). The advantage of this scenario is that it provides a natural explanation to a wide range of different phenomenologies commonly observed in X-ray bright AGN, such as the "soft excess" (Crummy et al. 2006) and the lack of response of relativistic reprocessed features to changes in the primary illuminating continuum (Miniutti \& Fabian 2004; Ponti et al. 2006). Does this scenario also hold for "less extreme", non-reflection-dominated AGN? Observationally, we confirm that the most likely driver of the variation in the $E W$ is the solid angle subtended by the accretion disc (Fig. 8).

Resonant trapping of fluorescent photons (Matt et al. 1993) can significantly suppress the $E W$ of the line emitted by mildly ionised gas $\left(\xi \sim a\right.$ few hundreds erg $\left.\mathrm{cm} \mathrm{s}^{-1}\right)$. Alternatively, in many objects of the FERO+GREDOS sample the measured strength of the disk reprocessing features implies a solid angle subtended by the disk of $<2 \pi$ (assuming isotropic primary emission). What is the ultimate physical driver of the lowerthan-standard "reflection fraction" in weak broad-line Seyferts? One possible way of decreasing the "effective solid angle" of the reflecting matter is that the innermost flow is so highly ionised that iron is fully stripped and neither fluorescence nor recombination lines can then be produced. The ionisation parameter of the disc is expected to increase very rapidly close to (and beyond) the ISCO (Reynolds \& Fabian 2008), ensuring that it rapidly becomes "radiatively truncated". The current data are insensitive to the detailed ionisation state of the relativistic line profiles. Although this prevents a conclusive answer on this possibility, there is no compelling evidence of a dependence of the line strength on the rest-frame centroid energy (de la Calle Pérez et al. 2010).

Beloborodov (1999) proposed that bulk motion of the primary X-ray continuum emitting plasma away from the accretion disc could explain the weakness of the reflection features. Reflection fractions corresponding to $\Omega<\pi$ can be achieved with moderate $(\beta \equiv v / c \simeq 0.3$ ) bulk velocities, and could be reduced further by the scattering of the reflected radiation in an outflowing blob. The X-ray outflowing plasma could be 
M. Guainazzi et al.: The ultimate driver of relativistic effects in AGN
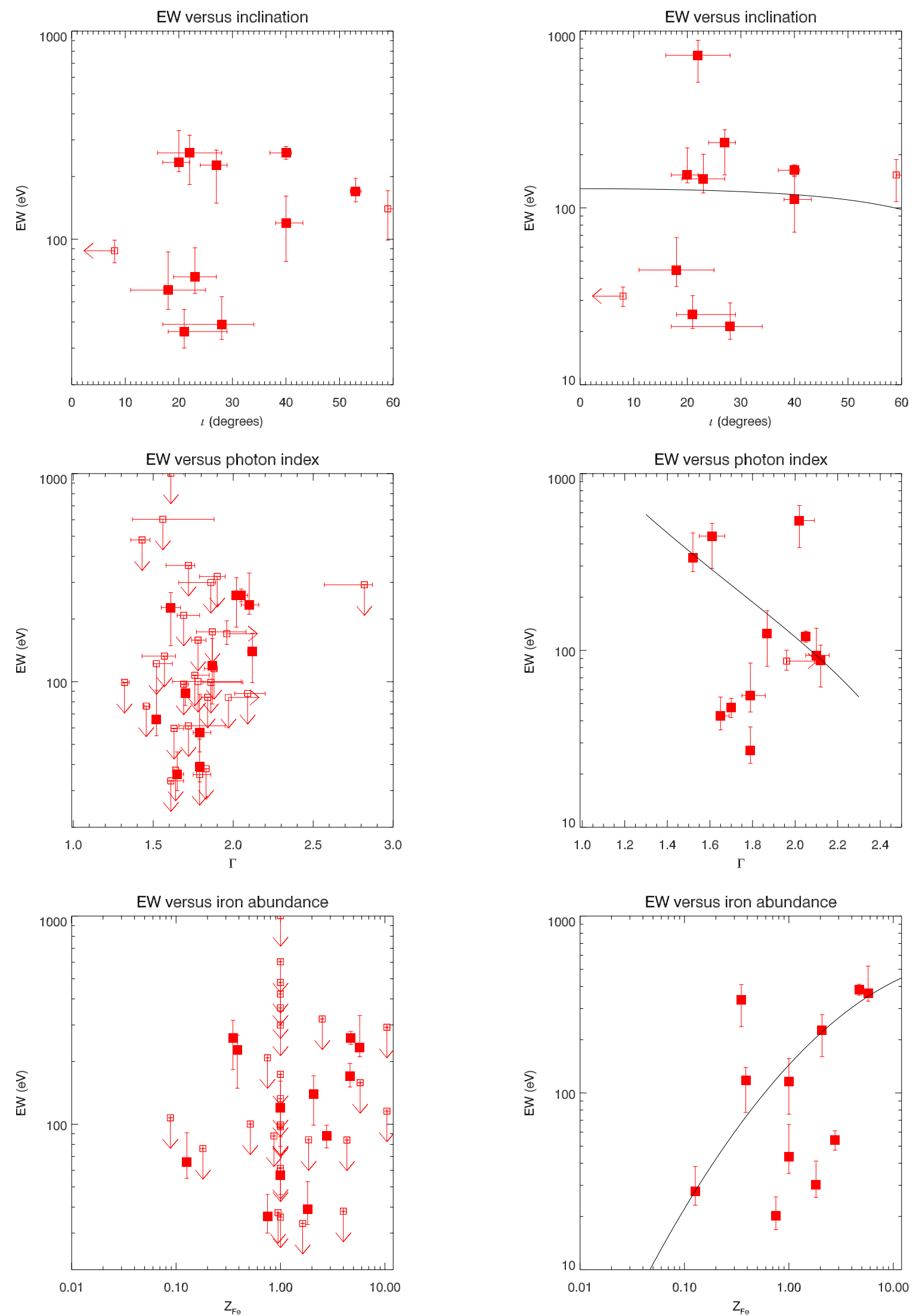

Fig. 7. $E W$ of the relativistically broadened iron line in the FERO+GREDOS sample against the: disc inclination angle (upper panels); primary illuminating continuum power-law spectral index (medium panels); and iron abundance (lower panels). The left column shows the whole sample, the right column shows only the data points corresponding to line detections, after correction of the $E W$ values for the dependence on the other two parameters according to George \& Fabian (1991) and using the following common reference values: $\Gamma_{0}=1.9 ; \iota_{0}=30^{\circ} ; Z_{\mathrm{Fe}, 0}=Z_{\odot}$. The solid line indicates the predicted behaviour according to George \& Fabian (1991). Filled symbols indicate detections. 

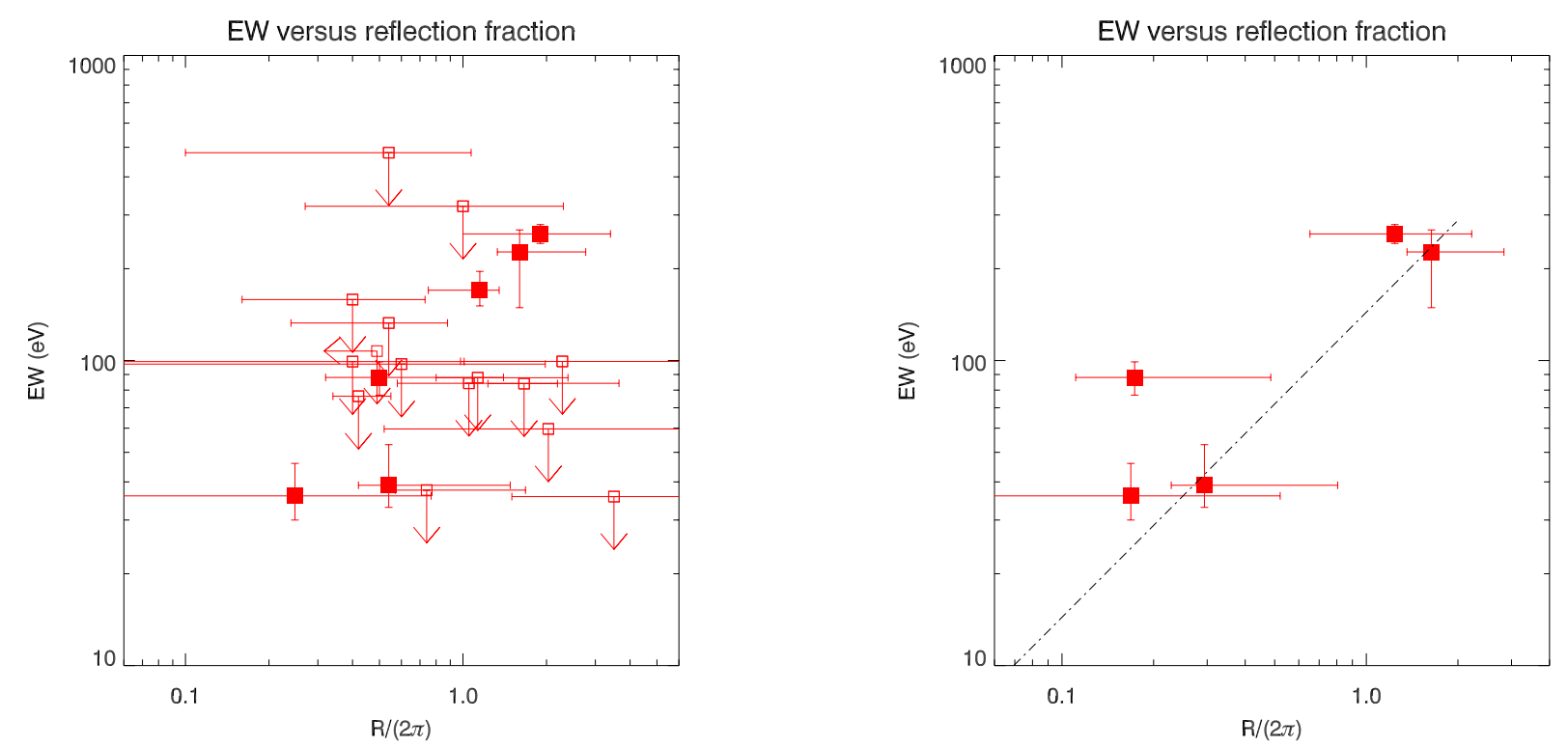

Fig. 8. EW against the "reflection fraction" parameter $R$ in the FERO+GREDOS sample. Left panel: all measurements. Right panel: detections only, corrected for the $E W$ dependences on $\Gamma, \imath$, and $Z_{\mathrm{Fe}}$ to the reference values of Fig. 7. The dashed line indicates a linear relation, with $E W=144 \mathrm{eV}$ for $R=1$ (George \& Fabian 1991).

identified with, e.g., the base of a jet, which would at the same time provide a source of the relativistic electrons required for inverse Compton upscattering of disc photons into the X-ray regime.

Alternatively, the disc could be physically truncated in sources with a smaller- $E W$ line. This mechanism would also provide a natural explanation of the correlation between the $E W$ and the power-law index of the disc emissivity as a function of radius (Fig. 5). In qualitative terms, a smaller $E W$ could be produced by a smaller area of the disc farther away from the black hole, where the relativistic effects are weaker; this would be reflected in the way we fit the data using low $(\alpha \leq 2)$ values. However, an alternative explanation of the same correlation invokes a simpler effect related to the quality of the data: when the red wing of a relativistic line is confused in the underlying continuum, the line profile appears less "relativistic" and at the same time weaker. Therefore, an observational bias could also produce the correlation in Fig. 5. Moreover, claims of truncated discs have been made for sources with very different value of accretion rate, from low-luminosity AGN to powerful quasars (Matt et al. 2005; Svoboda et al. 2010; Lobban et al. 2010). This is at odds with a similar scenario invoked to explain the hard/low state in galactic black hole candidates (GBHCs), typically associated with low accretion states (Fender et al. 2004). A possible way of reconciling this discrepancy (apart from invoking a different explanation for the lack of relativistic features in high accretion rate $\mathrm{AGN}$ ) is assuming that powerful quasars never go into the equivalent of the GBHC "low state". States without relativistic spectral features would correspond to the "very high state" observed, for instance, in the micro-quasar GRS1915+105 (Fender \& Belloni 2004).

The broad iron line could also be produced by localised co-rotating spots on the accretion disc, which illuminate only a small fraction thereof (Pecháček et al. 2005). Transient redshifted features have been indeed reported in an handful of objects (Turner et al. 2002; Guainazzi 2003) and interpreted in terms of orbiting spots (Dovčiak et al. 2004). Although doubts have been cast on the statistical significance of these effects
(Vaughan \& Uttley 2008), they have been confirmed by a homogeneous study of a large number of bright sources extracted from the FERO sample (de Marco et al. 2009). The reliability of this phenomenology and its relevance to the AGN population as a whole is still uncertain, and will hopefully be fully understood as more data become available with future high-throughput missions.

\subsection{On alternatives to the relativistic blurring scenarios}

Finally, if the spectral complexity of the iron band is due to a completely different mechanism, which does not incorporate general relativistic effects close to the innermost region of the accretion flow (e.g., Turner \& Miller 2009), the observations described in this paper cannot be explained in terms of relativistic effects. Turner \& Miller (2009) recently reviewed an alternative scenario where the skewed profile of the $\mathrm{K}_{\alpha}$ iron line is due to partial covering ionised absorption mimicking the broad red wing and the sharp blue drop at $\simeq 7 \mathrm{keV}$. This scenario delivers statistically equivalent fits to the relativistic reflection scenario for a number of time-averaged X-ray spectra of bright Seyferts (Reeves et al. 2004; Miller et al. 2009), as well as explaining their spectral variability (Miller et al. 2007, 2008, 2010). Readers are referred to Miller (2007) and Turner \& Miller (2009) for an exhaustive discussion of the strengths and weaknesses of both scenarios. Disk winds are predicted theoretically in several configurations of the accretion flow (Proga et al. 2008; Sim et al. 2010), and their impact on the spectral shape above $2 \mathrm{keV}$ cannot be neglected (Reeves et al. 2004). The authors of the current paper are of the opinion the complex geometry required by partial covering of the primary X-ray source on scales of a few gravitation radii (Risaliti et al. 2007) and the scale-law between GBH systems (where partial covering surely does not occur) and AGN in the timing-domain (McHardy et al. 2006) are serious shortcomings of the "partial covering" scenario. In this context, the possible discovery of "clean AGN" (i.e., AGN whose X-ray soft X-ray spectrum does not show evidence of a 
M. Guainazzi et al.: The ultimate driver of relativistic effects in AGN
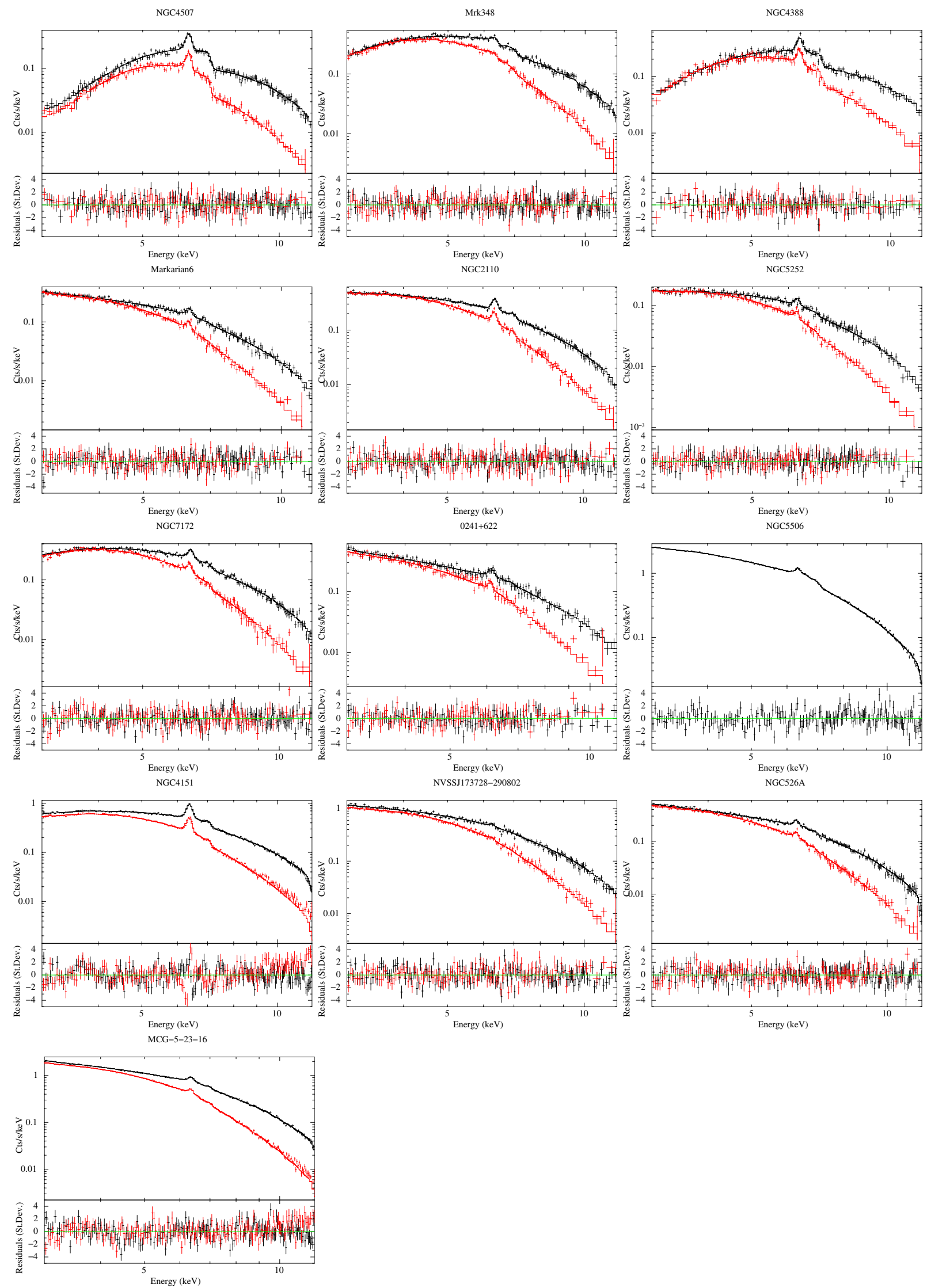

Fig. 9. Spectra (upper panels) and residuals in units of standard deviations (lower panels) when the best-fit models given in Table 3 are applied to the GREDOS sample. 
warm absorber; Emmanoulopoulos et al. 2011) are a potentially crucial testbed for the robustness of $\mathrm{Fe} \mathrm{K}_{\alpha}$ broad line detections.

Turner \& Miller (2009) and many papers referenced therein discuss these shortcomings. They stress that the light bending scenario - introduced to explain an unexpected lack of correlation between the short-term variability of the primary continuum and the disk reflection features - also requires rather "ad hoc" assumptions about the X-ray source geometry and dynamics. The source has to be compact and to move in a vertical direction on short timescales while maintaining its stability. While the base of a jet could in principle provide a physical location for this compact, highly dynamical source of high-energy photons, some difficulties of this picture were discussed by Życki et al. (2010). In the partial covering scenario, the physical scaling of the accreting system with mass and luminosity implies a scaling of the clumpy covering material with mass. While short-time variations at high energy are still most likely to be caused by intrinsic fluctuations of the source, the spectral curvature below the $\mathrm{Fe} \mathrm{K}_{\alpha}$ line and its variation on long timescales are successfully modelled with clumpy material in a disk wind.

It remains highly controversial which falsification experiment(s) could eventually settle the issues. General consensus exists that studies of large AGN X-ray high-quality broad-band spectral samples with operational (Suzaku; see, e.g., the recent work by Patrick et al. 2011) or future (Astro-H, Astrosat, IXO) missions will be crucial. A statistical comparison of the fit quality produced by different scenarios for the same sample, as well as of the corresponding observable distributions, would be a valuable exercise. This paper, the last of a series of studies inspired by the goal of putting the discussion on relativistic X-ray spectroscopy on a sound statistical basis, aims to contribute to this ongoing effort.

\section{Appendix A: Spectral results on the GREDOS sample}

Table 3 summarises the results for the continuum components.

In Table 4, we report the results for the relativistic line profiles. For non-detected lines, we report only the $90 \%$ upper limit to the $E W$ for a fixed $E_{\mathrm{c}} \equiv 6.4 \mathrm{keV}$. The parameters refers to a value of the spin corresponding to a maximally spinning black hole $(a=0.998)$. In all cases, a solution corresponding to a neutral emitting gas $\left(E_{\mathrm{c}}=6.4 \mathrm{keV}\right)$ is preferred. A gallery of spectra and best-fit model is shown in Fig. 9, whereas the line profiles (only for detections) are shown in Fig. 2. These line profiles are obtained from the data/model ratio against the best-fit model once the relativistic line profile (kyrline component in the best-fit model) is removed. In a few cases, the strength of the continuum reflection as measured by EPIC is inconsistent with the broad iron line $E W$, or lack thereof. We stress that fits of the EPIC-pn spectra are relatively insensitive to the exact value of the reflection fraction, which is primarily driven by the depth and shape of the iron photo-absorption edge. This feature is degenerate with the photo-absorption column density in fits of obscured AGN over an energy band limited to $10 \mathrm{keV}$. One should therefore be careful when inferring the astrophysical implications of these results.

Acknowledgements. This study has been based on observations obtained with $X M M-N e w t o n$, an ESA science mission with instruments and contributions directly funded by ESA Member States and NASA. This research has made use of the NASA/IPAC Extragalactic Database (NED) which is operated by the Jet Propulsion Laboratory, California Institute of Technology, under contract with the National Aeronautics and Space Administration. The authors thank G. Cusumano, V. La Parola, and G. Miniutti for comments on an early version of the manuscript. Constructive comments by the referee, Lance Miller, substantially improved the quality of the paper, allowing us to present our results in a more balanced and unbiased way.

\section{References}

Agol, E., \& Krolik, J. H. 2000, ApJ, 528, 161

Anders, E., \& Grevesse, N. 1989, Geochim. Cosmochim. Acta, 53, 197 Antonucci, R. 1993, ARA\&A, 31, 473

Awaki, H., Koyama, K., Inoue, H., \& Halpern, J. O. 1991, PASJ, 43, 195 Ballantyne, D. R. 2010, ApJ, 708, L1

Beckmann, V., Gehrels, N., Favre, P., et al. 2004, ApJ, 614, 641

Beloborodov, A. M. 1999, ApJ, 510, L123

Bennett, C. L., Halpern, M., Hinshaw, G., et al. 2003, ApJS, 148, 1

Bianchi, S., \& Matt, G. 2002, A\&A, 387, 76

Bianchi, S., Guainazzi, M., \& Chiaberge, M. 2006, A\&A, 448, 499

Bianchi, S., Guainazzi, M., Matt, G., \& Fonseca-Bonilla, N. 2007, A\&A, 467, L19

Bianchi, S., de Angelis, I., Matt, G., et al. 2010, A\&A, 522, A64

Branduardi-Raymont, G., Sako, M., Kahn, S. M., et al. 2001, A\&A, 365, L140

Brenneman, L. W., \& Reynolds, C. S. 2006, ApJ, 652, 1028

Chartas, G., Eracleous, M., Dai, X., Agol, E., \& Gallagher, S. 2007, ApJ, 661, 678

Crummy, J., Fabian, A. C., Gallo, L., \& Ross, R. R. 2006, MNRAS, 365, 1067

Cusumano, G., La Parola, V., Segreto, A., et al. 2010, A\&A, 510, A48

Dadina, M. 2007, A\&A, 461, 1209

Dadina, M. 2008, A\&A, 485, 417

Dadina, M., Guainazzi, M., Cappi, M., et al. 2010, A\&A, 516, A9

de la Calle Pérez, I., Longinotti, A., Guainazzi, M., et al. 2010, A\&A, 524, A50

Della Ceca, R., Ballo, L., Braito, V., \& Maccacaro, T. 2005, ApJ, 627, 706

de Marco, B., Iwasawa, K., Cappi, M., et al. 2009, A\&A, 507, 159

Dewangan, G. C., Griffiths, R. E., \& Schurch, N. E. 2003, ApJ, 592, 52

Dovčiak, M., Bianchi, S., Guainazzi, M., Karas, V., \& Matt, G. 2004, MNRAS, 350,745

Emmanoulopoulos, D., Papadakis, I. E., McHardy, I. M., et al. 2011, MNRAS, 408, 1928

Fabian, A. C., \& Vaughan, S. 2003, MNRAS, 340, L28

Fender, R. P., \& Belloni, T. M. 2004, ARA\&A, 42, 317

Fabian, A. C., Rees, M. J., Stella L., \& White, N. E. 1989, MNRAS, 238, 729

Fabian, A. C., Iwasawa, K., Reynolds, C. S., \& Young, A. J. 2000, PASP, 112 1145

Fabian, A. C., Vaughan, S., Nandra, K., et al. 2002, MNRAS, 335, L1

Fabian, A. C., Zoghbi, A., Ross, R. R., et al. 2009, Nature, 459, 540

Fender, R. P., Belloni, T. M., \& Gallo, E. 2004, MNRAS, 355, 1105

Ferland, G. J., Korista, K. T., Verner, D. A., et al. 1998, PASP, 110, 761

Gabriel, C., Denby, M., Fyfe, D. J., Hoar, J., \& Ibarra, A. 2003, in Astronomical

Data Analysis Software and Systems XIII, ed. F. Ochsenbein, M. Allen, \& D.

Egret (San Francisco: ASP), ASP Conf. Ser., 314, 759

George, I. M., \& Fabian, A. C. 1991, MNRAS, 249, 352

Guainazzi, M. 2003, A\&A, 401, 903

Guainazzi, M., Bianchi, S., \& Dovĉiak, M. 2006, AN, 327, 1032

Guainazzi, M., Risaliti, G., Nucita, A., et al. 2009, A\&A, 505, 589

Guainazzi, M., Bianchi, S., Matt, G., et al. 2010, MNRAS, 406, 2013

Isobe, T., Feigelson, E. D., \& Nelson, P. I. 1986, ApJ, 306, 490

Krolik, J. H., \& Hawley, J. F. 2002, ApJ, 573, 754

Krumpe, M., Lamer, G., Schwope, A., \& Husemann, B. 2007, A\&A, 470, 497

Lampton, M., Margon, B., \& Bowyer, S. 1976, ApJ, 207, 894

Laor, A. 1991, ApJ, 376, 90

Lobban, A. P., Reeves, J. N., Porquet, D., et al. 2010, MNRAS, 408, 551

Longinotti, A. L., Cappi, M., Nandra, K., Dadina, M., \& Pellegrini, S. 2003, A\&A, 410, 471

Longinotti, A. L., Bianchi, S., Santos L. M., et al. 2007, MNRAS, 374, 237

Magdziarz, P., \& Zdziarski, A. A. 1995, MNRAS, 273, 837

Markowitz, A., Reeves, J. N., \& Braito, V. 2006, ApJ, 646, 783

Markowitz, A., Reeves, J. N., Miniutti, G., et al. 2008, PASJ, 60, 277

Martocchia, A., Matt, G., \& Karas, V. 2002, 383, L23

Mason, K., Branduardi-Raymont, G., Ogle, P. M., et al. 2003, ApJ, 582, 95

Matt, G. 2002, MNRAS, 337147

Matt, G., Perola, G. C., Piro, L., \& Stella, L. 1992, A\&A, 257, 63 (erratum: 263, 453)

Matt, G., Fabian, A. C., \& Ross, R. R. 1993, MNRAS, 261, 346

Matt, G., Bianchi, S., d'Ammando, F., \& Martocchia, A. 2004, A\&A, 421, 473

Matt, G., Porquet, D., Bianchi, S., et al. 2005, A\&A, 435, 857

McHardy, I., Koerding, E., Knigge, C., Uttley, P., \& Fender, R. P. 2006, Nature, 444, 730

Miller, J. M. 2007, ARA\&A, 45, 441

Miller, L., \& Turner, T. J. 2010, MNRAS, 408, 1928 
M. Guainazzi et al.: The ultimate driver of relativistic effects in AGN

Miller, L., Turner, T. J., Reeves, J. N., et al. 2007, A\&A, 463, 131

Miller, L., Turner, T. J., \& Reeves, J. 2008, A\&A, 483, 437

Miller, L., Turner, T. J., \& Reeves, J. 2009, MNRAS, 399, L69

Miller, L., Turner, T. J., Reeves, J. N., et al. 2010, MNRAS, 403, 196

Miniutti, G., \& Fabian, A. C. 2004, MNRAS, 349, 1435

Nandra, K., \& Pounds, K. A. 1994, MNRAS, 268, 405

Nandra, K., George, I. M., Mushotzky, R. F., Turner, T. J., \& Yaqoob, T. 1997, ApJ, 467, 70

Nandra, K., O’Neill, P. M., George, I. M., \& Reeves, J. N. 2007, MNRAS, 382, 194

Nayakshin, S., Kazanas, D., \& Kallman, T. R. 2000, ApJ, 537, 833

Netzer, H., \& Trakhtenbrot, B. 2007, ApJ, 654, 754

Netzer, H., Turner, T. J., \& George, I. M. 1998, ApJ, 504, 680

Page, K. L., O’Brien, P. T., Reeves, J. N., \& Turner, M. J. L. 2004, MNRAS, 347, 316

Patrick, A. R., Reeves, J. N., Porquet, D., et al. 2011, MNRAS, 411, 2353

Pecháček, T., Dovčiak, M., Karas, V., \& Matt, G. 2005, A\&A, 441, 855

Perola, G. C., Matt, G., Cappi, M., et al. 2002, A\&A, 389, 202

Piconcelli, E., Sánchez Portal, M., Guainazzi, M., et al. 2006, A\&A, 453, 839

Ponti, G., Miniutti, G., Cappi, M., et al. 2006, MNRAS, 368, 903

Ponti, G., Fabian, A. C., Gallo, L. C., et al. 2010, MNRAS, 406, 2591

Porquet, D. 2006, A\&A, 445, L5

Pounds, K. A., Nandra, K., Stewart, G. C., George, I. M., \& Fabian, A. C. 1990, Nature, 344, 132

Proga, D., Ostriker, J. P., \& Kurosawa, R. 2008, ApJ, 676, 101

Reeves, J. N., Nandra, K., George, I. M., et al. 2004, ApJ, 602, 648

Reeves, J. N., Awaki, H., Dewangan, G. C., et al. 2007, PASJ, 59, 301

Reeves, J. N., O'Brien, P., Braito, V., et al. 2009, ApJ, 701, 493

Reynolds, C. S., \& Fabian, A. C. 2008, ApJ, 675, 1048

Reynolds, C. S., \& Novak, M. A. 2003, PhR, 377, 389

Reynolds, C. S., Wilms, J., Begelman, M. C., Staubert, R., \& Kendziorra, E. 2004, MNRAS, 349, 1153
Revnivtsev, M., Sazonov, S., Jahoda, K., \& Gilfanov, M. 2004, A\&A, 418, 927 Risaliti, G. 2002, A\&A, 386, 379

Risaliti, G., Elvis, M., Fabbiano, G., et al. 2007, ApJ, 659, L111

Risaliti, G., Miniutti, G., Elvis, M., et al. 2009, ApJ, 696, 160

Ross, R. R., \& Fabian, A. C. 2005, MNRAS, 358, 211

Schmitt, J. H. M. M. 1985, A\&A, 293, 178

Schurch, N. J., Warwick, R. S., Griffiths, R. E., \& Sembay, S. 2003, MNRAS, 345,423

Schurch, N. J., Griffiths, R. E., \& Warwick, R. S. 2006, MNRAS, 317, 211

Shu, X. W., Yaqoob, T., \& Wang, J. X. 2010, ApJS, 187, 581

Sim, S. A., Proga, D., Miller, L., Long, K. S., \& Turner, T. J. 2010, MNRAS, 408, 1386

Strüder, L., Briel, U., Dannerl, K., et al. 2001, A\&A, 365, L18

Svoboda, J., Guainazzi, M., \& Karas, V. 2010, A\&A, 512, A62

Tanaka, Y., Nandra, K., Fabian, A. C., et al. 1995, Nature, 375, 659

Turner, T. J., George, I. M., Nandra, K., \& Mushotzky, R. F. 1997, ApJS, 113 23

Turner, M. J. L., Abbey, A., Arnaud, M., et al. 2001, A\&A, 365, L27

Turner, T. J., \& Miller, L. 2009, A\&ARv, 17, 47

Turner, T. J., Miller, L., Kraemer, S. B., Reeves, J. N., \& Pounds, K. A. 2009 ApJ, 698, 99

Turner, T. J., Mushotzky, R. F., Yaqoob, T., et al. 2002, ApJ, 574, L12

Vaughan, S., \& Uttley, P. 2008, MNRAS, 390, 412

Véron-Cetty, M.-P., \& Véron, P. 2010, A\&A, 518, A10

Vignali, C., Piconcelli, E., Bianchi, S., \& Miniutti, G. 2008, MNRAS, 388, 761

Walton, D. J., Reis, R. C., \& Fabian, A. C. 2010, MNRAS, 408, 601

Wilms, J., Reynolds, C. S., Begelman, M. C., et al. 2001, MNRAS, 328, L27

Życki, P., Krolik, J. H., Zdziarski, A. A., \& Kallman, T. R. 1994, ApJ, 437, 596

Życki, P., Ebisawa, K., Niedźwiecki, A., \& Miyakawa, T. 2010, PASJ, 62, 1185 